\title{
LEITOS PSIQUIÁTRICOS EM HOSPITAL GERAL: VISÃO DE PROFISSIONAIS QUE ATUAM EM HOSPITAL GERAL
}

\author{
PSYCHIATRIC WARD IN GENERAL HOSPITAL: PROFESSIONALS VIEW WHO WORKS IN GENERAL HOSPITAL \\ LOS LECHOS PSIQUIÁTRICOS EN HOSPITAL GENERAL: VISIÓN DE LOS PROFESIONALES QUE ACTUAN EN \\ HOSPITAL GENERAL
}

Jucéli Zimmermann Mion ${ }^{1}$ Jacó Fernando Schneider ${ }^{2}$

RESUMO: A proposta deste estudo foi a de buscar junto aos profissionais de saúde que atuam em hospital geral, a percepção e a aceitação dos mesmos sobre a possível implantação de leitos psiquiátricos na instituição em que atuam. A pesquisa foi realizada em cinco hospitais gerais privados da cidade de Cascavel, Estado do Paraná, que prestam assistência não só a pacientes do município mas da região oeste do estado.

PALAVRAS ChAVES: Psiquiatria, Leitos Psiquiátricos, Hospital Geral, Saúde Mental

ABSTRACT: The proposal of this study was the one of looking for the professionals of health that act in general hospital, the perception and the acceptance of the same ones on the possible implantation of psychiatric beds in the institution close to in that act. The research was accomplished at five private general hospitals of the city of Cascavel, State of Paraná, that you/they render attendance not only to patient of the municipal district but of every west area of the state of Paraná.

KEY WORDS: Psychiatry, Psychiatric Beds, General Hospital, Mental Health

RESUMEN: La propuesta de este estudio fue de coger junto a los profesionales de la salud que actuan en hospital general, la percepción y la aceptación de los mismos a cerca de la posible implantación de lechos psiquiátricos en la institución donde actuan. La pesquisa fue realizada en cinco hospitales privados de la ciudad de Cascavel, en Estado de Paraná, que prestan asistencia no sólo a pacientes del municipio, pero de la región oeste del Estado.

TERMINOS CLAVES: Psiquiatria, Lechos Psiquiátricos, Hospital General, Salud Mental

\section{INTRODUÇÃO}

Durante nossa trajetória como profissionais de enfermagem são tantas as discussões sobre a Reforma Psiquiátrica, a desconstrução do aparato manicomial, criação de unidades psiquiátricas em hospitais gerais, que nos despertaram para a necessidade de saber como essas medidas são vistas e qual a aceitação dos profissionais da rede hospitalar privada, no que diz respeito a essas mudanças.

Para tanto, os participantes desta pesquisa foram os profissionais que trabalham em hospital geral: diretores, médicos, enfermeiros, auxiliares de enfermagem, copeiras e serventes de limpeza, ou seja, as pessoas que terão contato com esses pacientes, se a Reforma Psiquiátrica vier a se concretizar, com a criação de Leitos Psiquiátricos em Hospitais Gerais.

Assim, o objetivo deste estudo foi o de buscar junto aos profissionais de saúde que atuam em hospital geral, a percepção e a aceitação dos mesmos sobre a possível implantação de leitos psiquiátricos na instituição em que atuam.

\section{MATERIAL E MÉTODO}

A busca do conhecimento pode ser feita de diversas maneiras e essa diversidade se dá ao fato de que o ato de conhecer é próprio do homem. O ser humano ao enfrentar os problemas procura identificar soluções e neste enfrentamento, produz conhecimentos. Minayo (1999: 12) fala que:

... o labor científico caminha sempre em duas direções: numa, elabora suas teorias, seus métodos, seus princípios e estabelece seus resultados; noutra, inventa, ratifica seu caminho, abandona certas vias e encaminhase para certas direções previlegiadas. $E$ ao fazer tal percurso, os investigadores aceitam os critérios da historicidade, da colaboração e, sobretudo, imbuem-se da humildade de quem sabe que qualquer conhecimento é aproximado, é construído.

A pesquisa científica objetiva responder questões, buscar soluções para os problemas. O método científico é o instrumento para auxiliar a compreender o mundo, portanto é o caminho para aquisição do conhecimento.

Por meio de métodos científicos, os pesquisadores lutam para a solução de problemas, para dar sentido à experiência humana, para compreender a regularidade dos fenômenos e para prever circunstâncias futuras. Mas, o que existe de tão especial no método científico? Consciente ou

\footnotetext{
${ }^{1}$ Enfermeira. Especialista em Saúde Pública. Secretaria Municipal de Saúde de Cafelândia, Paraná.

2 Enfermeiro. Doutor em Enfermagem. Docente do Colegiado do Curso de Enfermagem da Universidade Estadual do Oeste do Paraná. E-mail: jaco@unioeste.br
} 
inconscientemente, nós todos fazemos indagações, resolvemos problemas e tomamos decisões, a cada dia (POLIT; HUNGLER, 1995: 12).

No entanto, todo pesquisador precisa seguir uma metodologia, garantindo assim, percorrer um caminho sistemático e ordenado, evitando através dela, tirar conclusões baseadas na intuição.

Sobre pesquisa científica, Martins e Bicudo (1989: 65) colocam que: "Toda pesquisa científica, pressupõe sempre uma posição, uma postura que torna possível investigar os fenômenos, a partir de uma certa perspectiva, na qual habilita o pesquisador a encontrar resposta para sua problemática".

$\mathrm{Na}$ pesquisa científica, existem dois métodos: a pesquisa quantitativa que trabalha com fatos, dados estatísticos, números, e a pesquisa qualitativa que trabalha com dados subjetivos, crenças, valores, opiniões, fenômenos, hábitos.

Como nossa problemática estava voltada à percepção, ou seja, conhecer como os profissiobnais de saúde de hospitais gerais privados percebem a possibilidade de implantação de leitos psiquiátricos nas instituições em que trabalham, usamos como referencial a pesquisa qualitativa, que consideramos ser a mais apropriada.

Sobre a pesquisa qualitativa, Minayo (1999: 21) coloca que:

Ela se preocupa, nas ciências sociais, com um nível de realidade que não pode ser quantificado. Ou seja, ela trabalha com um universo de significados, motivos, aspirações, crenças, valores e atitudes, o que corresponde a um espaço mais profundo das relações, dos processos e dos fenômenos que não podem ser reduzidos à operacionalização de variáveis.

$\mathrm{Na}$ pesquisa qualitativa, o material utilizado é a palavra, que expressa a fala cotidiana, nos discursos intelectuais, burocráticos, políticos e nas relações afetivas ou técnicas. Neste método, procura-se interpretar o conteúdo das falas, ultrapassando a mensagem e conhecendo significados latentes.

Ainda sobre o método qualitativo, Minayo (1999:

24) enfatiza que:

Os autores que seguem tal corrente não se preocupam em quantificar, mas, sim, compreender e explicar a dinâmica das relações sociais que, por sua vez, são depositárias de crenças, valores, atitudes e hábitos. Trabalham com a vivência, com a experiência, com a continuidade e também com a compreensão das estruturas e instituições como resultado da ação humana objetiva. Ou seja, desse ponto de vista, a linguagem, as práticas $e$ as coisas são inseparáveis.

Assim, nesta investigação, os dados foram coletados através de uma entrevista aberta, pois pensamos ser este o instrumento mais apropriado para a pesquisa qualitativa.

Sobre este recurso, Gil (1987: 113) coloca que:
"Pode-se definir como a técnica em que o investigador se apresenta frente ao investigado e Ihe formúla perguntas, com o objetivo de obtenção dos dados que interessam à investigação.

A entrevista é portanto, uma forma de interação social. Mais especificamente, é uma forma de diálogo assimétrico, em que uma das partes busca coletar dados e a outra se apresenta como fonte de informação".

$\mathrm{Na}$ operacionalização deste estudo, observou-se as normas éticas no que se refere à pesquisa envolvendo seres humanos, conforme preconizado na resolução 196/96 do Conselho Nacional de Saúde. Sendo assim, o projeto de pesquisa passou por um Comitê de Ética em Pesquisa com Seres Humanos e foi aprovado.

Anteriormente à pesquisa, foram procurados os diretores gerais destas instituições, para entrega de ofício, solicitando campo de pesquisa. Todos concordaram dando autorização no próprio ofício.

Assim, a pesquisa foi realizada em cinco hospitais gerais privados da cidade de Cascavel, Estado do Paraná, que prestam assistência não só a pacientes do município mas de toda região oeste do estado do Paraná.

A pesquisa foi realizada apenas nas instituições privadas por serem em maior número na cidade e, por poderem retratar na medida dos objetivos que traçamos a percepção dos profissionais que atuam nos hospitais. Por ter apenas um hospital público no município onde o estudo foi desenvolvido, pensamos que se tornaria difícil estabelecer comparações entre as percepções dos profissionais da área pública e da área privada, sendo necessária outra investigação para se atingir este objetivo.

Foram entrevistados diretores gerais, médicos, enfermeiros, auxiliares de enfermagem, copeiras e serventes de limpeza. A escolha destas categorias deu-se ao fato de que se houver implantação de leitos psiquiátricos em hospitais gerais privados, estes profissionais terão contato com os pacientes psiquiátricos.

As entrevistas foram realizadas por um dos pesquisadores dentro dos hospitais, em horário de trabalho e os profissionais entrevistados foram os que estavam de plantão. Foram realizadas em dias e horários diferentes, conforme disponibilidade dos respondentes.

Todos os entrevistados foram inteirados individualmente da pesquisa e, que esta seria realizada através de entrevista gravada. Foi feita uma única pergunta, com a seguinte questão norteadora: "Qual a sua percepção frente à possibilidade de implantação de leitos psiquiátricos neste hospital?".

Os dados coletados foram analisados tendo como referencial a análise de conteúdo proposta por Bardin, (1977) apud Gil (1987:163-4), que apresenta os seguintes passos: a) pré-análise; b) exploração do material; e c) tratamento dos dados, inferência e interpretação. 
Após a transcrição na íntegra das falas foi efetuada análise dos conteúdos, que resultou em seis (6) unidades temáticas. Esta análise "(...) consiste em descobrir os 'núcleos de sentido' que compõem a comunicação e cuja presença, ou freqüência de aparição podem significar alguma coisa para o objectivo analítico escolhido" (BARDIN, 1977:105).

A seguir, apresentaremos as unidades temáticas para análise dos discursos, como segue: A implantação de leitos psiquiátricos em hospital geral está condicionada a infra-estrutura do hospital; A implantação de leitos psiquiátricos em hospital geral está vinculada à qualificação da equipe que irá atuar junto a pacientes em sofrimento psíquico; A implantação de leitos psiquiátricos em hospital geral vai favorecer a recuperação e reintegração mais rápida do indivíduo em sofrimento psíquico à sociedade; $\mathrm{A}$ implantação de leitos psiquiátricos em hospital geral está relacionada ao preconceito, ao estigma com relação ao portador de transtorno mental; A implantação de leitos psiquiátricos em hospital geral está ligada à questão financeira; A implantação de leitos psiquiátricos em hospital geral requer envolvimento da família dos pacientes psiquiátricos.

\section{RESULTADOS E DISCUSSÃO}

A análise dos conteúdos dos discursos foi embasada nas temáticas descritas acima, onde pretendeu-se discutir e relacionar os conteúdos com referenciais de autores que estudaram estas questões.

$\mathrm{Na}$ primeira unidade temática, aparece nos discursos a preocupação no sentido de organizar o espaço físico do hospital geral, para poder atender pacientes psiquiátricos.

Esta preocupação fundamenta-se no conhecimento dos profissionais de que estes pacientes precisam de um atendimento diferenciado, pois como se sentem bem fisicamente, permanecem pouco no leito. $\mathrm{O}$ ambiente físico adequado para portadores de patologias psíquicas, deve favorecer a orientação no tempo e no espaço (tendo relógios, calendários, espelhos, quadros) ter um refeitório, já que não se alimentam no leito, uma área de lazer para que possam participar de atividades físicas, sala de estar com rádio e televisão e que seja em andar térreo, facilitando o acesso para área de lazer e refeitório (BOTEGA, 1995).

Pensamos que a questão do espaço físico dificulta a implantação destes leitos, pois esta reorganização para adequar os hospitais está vinculada à disponibilidade de espaço físico e, implica em investimentos financeiros.

Os conteúdos da segunda unidade, que tem como temática a qualificação profissional, mostram que os profissionais não se sentem preparados para atender a clientela com transtorno mental. Esta questão era percebida enquanto enfermeira de hospital geral, onde a equipe multidisciplinar ao deparar-se com paciente portador de patologia psíquica, não se sentia à vontade para lidar com esta clientela, e estas falas evidenciam que o despreparo da equipe constitui-se em um problema para a implantação de leitos psiquiátricos em hospital geral.

Todos os discursos falam que seria viável a implantação destes leitos, desde que houvesse treinamentos e reciclagem para a equipe que atuaria junto ao doente.

Com relação ao pessoal auxiliar, Peduzzi (1992: 68) explica que:

Ao pensarmos na capacitação de pessoal auxiliar na área de saúde mental, tomamos como questão nuclear à interação entre subjetividade, pois que esta é a ferramenta básica com a qual os auxiliares realizarão seu trabalho de assistência à clientela. Para desenvolver tal habilidade terão que, primeiramente, adquirir um dado conhecimento através de ensino do saber específico da área. Os princípios e conceitos gerais que fundamentam o trabalho na instituição e, mais especificamente, na equipe de saúde mental serão os mesmos para o conjunto dos trabalhadores, diferindo a forma e a extensão em que serão transmitidos, e o modo e objetivos com que serão utilizados.

As referências dos participantes sobre o problema do despreparo dos profissionais para assistir o doente mental, são compreensíveis à medida que até o momento a formação oferecida pelos cursos da área da saúde não está voltada para a capacitação destes profissionais, para prestar assistência em outro espaço a não ser o hospital psiquiátrico.

Entendendo que a questão da assistência ao ser humano em sofrimento psíquico requer que se construam outros espaços de atenção, como no caso - hospital geral, a formação/capacitação dos profissionais há que ser repensada, e os profissionais que já estão atuando na área deverão ser capacitados.

De modo geral, os profissionais acreditam que o internamento do paciente portador de sofrimento psíquico em hospital geral favorecerá a recuperação e a reintegração mais rápida do doente a sociedade, uma vez que o paciente não perde o vínculo com a realidade e na alta hospitalar, sai sem o rótulo, sem o estigma de internação em hospital psiquiátrico, conforme nos mostra a terceira unidade temática identificada nos discursos.

Falando sobre as principais vantagens das Unidades Psiquiátricas em Hospital Geral, Botega e Dalgalarrondo (1997: 50) pontuam que se ocorresse a "(...) diminuição do estigma da doença mental: o doente mental no hospital geral passaria a ser visto como um doente semelhante aos outros. Os hospitais psiquiátricos tradicionais, ao contrário, tenderiam a reforçar tal estigma".

A quarta unidade retrata o preconceito. Apesar dos profissionais entrevistados reconhecerem os benefícios advindos com a internação do paciente 
psiquiátrico em hospital geral, as falas evidenciam o preconceito em relação a estes pacientes.

$\mathrm{Na}$ tentativa de não assumir este preconceito, alguns colocam que haveria atritos com os pacientes diferenciados (particulares e de convênios), que são mais exigentes que os pacientes do SUS e não aceitariam ter próximo do seu quarto, pacientes com patologias psíquicas.

Uma questão que fica explícita nestes depoimentos é a vinculação direta entre doença mental e pobreza, revelando ainda as discrepâncias na assistência à saúde, em que não se pode ter, convivendo num mesmo espaço, pessoas de diferentes níveis sociais. A inclusão de serviços de psiquiatria em hospital geral pelo que se pode observar ocorreria com uma certa resistência. Um dos fatores citados como dificultadores deste processo é o preconceito.

Para muitos ainda, 'lugar de louco é no hospício'. Há o temor de que os doentes mentais possam representar um perigo de agressão fisica para os outros doentes do hospital e pessoal técnico. Tais temores não se sustentam perante as evidências de mais de cinqüenta anos de UIPHGs nos paises desenvolvidos (BOTEGA; DALGALARRONDO, 1997: 49).

$\mathrm{Na}$ quinta unidade, emergiu a questão dos recursos financeiros como importantes para a realização desta proposta. O hospital teria que investir em recursos humanos e na reestruturação da área física. Como a maior demanda é de pacientes usuários do Sistema Único de Saúde, questionam se haveria retorno para cobrir estes investimentos, que não seriam poucos.

Com relação a estes questionamentos que surgiram nas falas dos entrevistados, transparecendo receio quanto ao financiamento deste tipo de serviço, nos embasamos novamente em Botega e Dalgalarrondo (1997: 50) que refletem sobre esta questão, mais particularmente sobre problemas relacionados a formas de pagamentos, colocando que: "(...) em vários países, muitos seguros médicos e sistemas previdenciários têm uma política clara de pagar menos aos leitos psiquiátricos; a maioria dos seguros médicos privados no Brasil não cobre assistência psiquiátrica".

Na Sexta e última unidade temática, surge a questão do envolvimento da família dos pacientes psíquiátricos como um fator fundamental para a implantação de leitos psiquiátricos em hospital geral.

Nos discursos dos entrevistados emerge a família como algo contraditório. Alguns colocam a importância do envolvimento familiar no processo de tratamento do enfermo mentalmente, alegando que o internamento em hospital geral pode favorecer 0 acompanhamento por parte da família no processo de tratamento.

No entanto, outros dizem que a família não participa da hospitalização, abandonando o familiar, deixando sob responsabilidade da instituição os cuidados e decisões sobre o paciente.

Observamos que ocorre uma preocupação entre os entrevistados de que os indivíduos em sofrimento psíquico sejam abandonados pelos familiares, deixados por longo tempo nos hospitais gerais, caso ocorra a implantação de leitos psiquiátricos nestas instituições, repetindo-se assim, o modelo asilar que tanto contribuiu para a cronificação desses indivíduos, conforme nos mostra a história da psiquiatria e da saúde mental.

\section{ALGUMAS CONSIDERAÇÕES SOBRE O ESTUDO}

A maior dificuldade para a implantação dos leitos psiquiátricos em hospital geral privado está vinculada principalmente à questão financeira. Para a realização de tal propósito, as instituições teriam que reestruturar a área física do hospital, investir na capacitação dos profissionais já vinculados à empresa, bem como a contratação de novos profissionais que ainda não fazem parte da equipe como assistente social, psicólogo e terapeuta ocupacional.

É de conhecimento de todos a insatisfação dos profissionais e dos serviços médico-hospitalar em relação ao pagamento dos serviços prestados ao Sistema Único de Saúde (SUS).

Sendo assim, quem arcará com os custos para o investimento necessário para a implantação de leitos psiquiátricos em hospital geral? Acredita-se que uma mudança desta natureza implica em mudança nas políticas de saúde e do modelo de assistência.

Além deste aspecto, não temos dúvida que qualquer modificação institucional somente ocorrerá se houver uma idéia global de mudança, incluindo todos os setores do hospital geral, dentre os quais a enfermagem, a medicina, a limpeza, a nutrição, o administrativo, enfim, todos os setores que se envolverão com o paciente psiquiátrico.

No entanto, além de levarmos em conta os aspectos citados, devemos considerar, como nos aponta Pitta (1993: 128), que existem infinitas dimensões de qualidade de serviços, encontradas numa tipologia de qualidade que podem ser úteis na implantação de leitos psiquiátricos em um hospital geral:

$1^{\circ}$ poderíamos iniciar falando sobre a qualidade social, que se mede em função da acessibilidade à prestação, ou seja, ao efetivo tratamento com as suas características de escuta, acolhida, negociação terapêutica. Entretanto, deve-se considerar não só de um ponto de vista da organização dos serviços mas também considerar a dimensão do custo para o usuário (econômico, afetivo e cultural). De um outro modo, um serviço pode ser definido com base na sua qualidade social não somente avaliando-se o grau de coerência entre o que o serviço oferece e o que o 
usuário demanda; a qualidade social se pode medir também em termos da aceitação do que se oferta nos caminhos da 'satisfação' do usuário. $2 q$ a qualidade econômica, definível em termos de eficiência, mensurável na relação entre custo e serviço ofertado. $3 q$ a qualidade técnica, mensurável em termos da correta aplicação do conhecimento e recurso humano e material disponível.

Isso se efetivando, os hospitais gerais terão condições concretas para a implantação com qualidade de leitos psiquiátricos, visto que este estudo mostrou que o pessoal técnico demonstra uma boa receptividade com relação a esta possibilidade.

Assim, realizar este trabalho de pesquisa, nos proporcionou além de um aprofundamento sobre a percepção dos profissionais de saúde frente à possibilidade de implantação de leitos psiquiátricos em hospital geral, uma maior reflexão sobre a condição do doente mental.

Neste sentido, percebemos que grande parte da problemática do sofrimento psíquico tem relação com o papel do ser humano na sociedade, visto que geralmente são indivíduos pouco produtivos que geram transtornos para as pessoas com as quais convivem.

Esta realidade é evidenciada mais entre as pessoas de baixo poder aquisitivo que, diante das dificuldades encontradas no convívio com um familiar em sofrimento mental, encontram no asilamento a solução de seus problemas, existindo convergência de interesses, pois para as instituições manicomiais este internamento também é conveniente.

Diante deste panorama, pensamos que as mudanças não podem acontecer apenas nos serviços de saúde, mas em toda a estrutura social e, que a dificuldade de implantação de formas alternativas de tratamento psiquiátrico, está ligada à forma de direcionamento dos recursos públicos para este tipo de assistência, já que a internação em instituição total ainda hoje é fonte segura de lucro para seus donos.

\section{REFERÊNCIAS BIBLIOGRÁFICAS}

BARDIN, L. Análise de conteúdo. Lisboa: Edições 70, 1977.

BOTEGA, N.J.(org.) Serviços de saúde mental no hospital geral. Campinas: Papirus, 1995.

; DALGALARRONDO, P. Saúde mental no

hospital geral: espaço para o psíquico. 2. ed. São Paulo: Hucitec, 1997.

GIL, A.C. Métodos e técnicas de pesquisa social. São Paulo: Atlas, 1987.

MINAYO, M.C.S. (Org.) Pesquisa social: teoria, método e criatividade. 13. ed. Petrópolis: Vozes, 1999. PEDUZZI. M. Capacitação de pessoal auxiliar em saúde mental: reflexões a partir da experiência de um centro de saúde escola. Saúde em debate. Santa Maria. n. 36, p. 66-72, out., 1992.
PITTA, A. Avaliando as transformações na assistência psiquiátrica brasileira. In: RUSSO, J.; SILVA FILHO, J.F. da (orgs.) Duzentos anos de psiquiatria. Rio de Janeiro: Relume Dumará/UFRJ, 1993. p. 125-34.

POLIT, D.F.; HUNGLER, B.P. Fundamentos de pesquisa em enfermagem. 3. ed. Porto Alegre: Artes Médicas, 1995.

Texto original recebido em 27/02/2003

Publicação aprovada em 27/06/2003 